\title{
JORDAN ALGEBRAS OF SELF-ADJOINT OPERATORS
}

\author{
BY \\ EDWARD G. EFFROS AND ERLING STØRMER
}

1. Introduction. A Jordan algebra of self-adjoint operators on a Hilbert space, or simply, a $J$-algebra, is a real linear space of such operators closed under the product $A \circ B=\frac{1}{2}(A B+B A)$. A $J C$-algebra, respectively, a $J W$-algebra, is a uniformly closed, respectively, weakly closed $J$-algebra (we show in $\S 3$ that $\sigma$-weakly closed $J$-algebras are weakly closed). In a recent paper [4], D. Topping has shown that many of the techniques used in the study of self-adjoint algebras of operators are applicable to $J$-algebras. We continue in this direction, proving that various problems are simplified by passing to the second dual.

We begin by showing that the second dual $\mathfrak{A}^{* *}$ of a $J$-algebra $\mathfrak{A}$ is isometric to a $J W$-algebra. We then use $\mathfrak{A}^{* *}$, together with the second dual of the $C^{*}$ algebra $[\mathfrak{A}]$ generated by $\mathfrak{A}$ to investigate the uniformly closed Jordan ideals in $\mathfrak{A}$. If $\mathfrak{\Im}$ is such an ideal we prove that $\mathfrak{A} / \mathfrak{\Im}$ is isometrically isomorphic to a $J$-algebra. We also show that if [ঞ] is the $C^{*}$-algebra generated by $\mathfrak{\Im}$, then $\mathfrak{\Im}=[\mathfrak{\Im}] \cap \mathfrak{A}$,

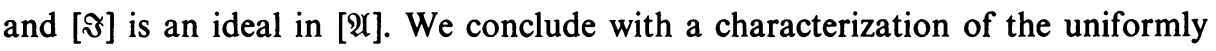
closed Jordan ideals in $\mathfrak{A}$. The simplified proof of this result was suggested to us by J. Ringrose.

We are indebted to D. Topping for invaluable correspondence on the subject.

2. Topological preliminaries. We recall that if $\mathfrak{X}$ is a real or complex normed linear space, there is a canonical isometry of $\mathfrak{X}$ into the second dual $\mathfrak{X}^{* *}$, sending the weak (i.e., $\sigma\left(\mathfrak{X}, \mathfrak{X}^{*}\right)$ ) topology into the relative weak* (i.e., $\sigma\left(\mathfrak{X}^{* *}, \mathfrak{X}^{*}\right)$ ) topology. We shall identify $\mathfrak{X}$ with its image. If $\mathfrak{A}$ is a uniformly closed convex subset of $\mathfrak{X}$, then it is weakly closed (see [2, p. 67]). It follows that if $\bar{\AA}$ is the weak* closure of $\mathfrak{\Omega}$ in $\mathfrak{X}^{* *}, \mathfrak{I}=\overline{\mathfrak{\Re}} \cap \mathfrak{X}$. If $\mathfrak{L}$ is a convex set in $\mathfrak{X}^{* *}$ containing 0 , the double polar $\mathfrak{L}^{00}$ coincides with the weak* closure $\overline{\mathbf{Q}}$.

We shall use a subscript 1 (resp., 0 ) to indicate the closed (resp., open) unit ball of a given normed linear space.

If $\mathfrak{X}$ and $\mathfrak{Y}$ are normed linear spaces, and $S$ is a linear isometry of $\mathfrak{X}$ into $\mathfrak{Y}$, the adjoint $S^{*}$ maps $\mathfrak{Y}_{1}^{*}$ onto $\mathfrak{X}_{1}^{*}$, and kernel $S^{*}=S(\mathfrak{X})^{0}$. On the other hand, if $T: \mathfrak{X} \rightarrow \mathfrak{Y}$ is a linear map with $T\left(\mathfrak{X}_{0}\right)=\mathfrak{Y}_{0}, T^{*}$ is an isometry and a weak* homeomorphism (see [2, p. 101]) of $\mathfrak{Y}^{*}$ into $\mathfrak{X}^{*}$, and $T^{*}\left(\mathfrak{Y}^{*}\right)=(\operatorname{kernel} T)^{0}$. Turning to the second adjoints, $S^{* *}$ is an isometry and weak* homeomorphism of $\mathfrak{X}^{* *}$ into $\mathfrak{Y}^{* *}$, extending $S$, and $S^{* *}\left(\mathfrak{X}^{* *}\right)=S(\mathfrak{X})^{00}=S(\mathfrak{X})^{-}$. Similarly, $T^{* *}\left(\mathfrak{X}_{1}^{* *}\right)=\mathfrak{Y}_{1}^{* *}$, and if $\mathfrak{I}=$ kernel $T$, kernel $T^{* *}=\mathfrak{\Re}^{00}=\overline{\mathfrak{A}} . T^{* *}$ induces an isometry of $\mathfrak{X}^{* *} / \overline{\mathfrak{\Re}}$ onto $\mathfrak{Y}^{* *}$.

Received by the editors October 31, 1965. 


\section{The second dual.}

THEOREM 1. Let $\mathfrak{A}$ be a J-algebra. There is an isometry of $\mathfrak{A} * *$ onto a JW-algebra, carrying the weak* topology onto the weak operator topology, and providing $\mathfrak{A}^{* *}$ with a multiplication extending that of $\mathfrak{A}$.

Proof. Let $\mathbb{E}$ be the $C^{*}$-algebra [ㅈ] . Due to the Sherman, Takeda, Grothendieck theory (see [3]), we may identify $\mathfrak{C}^{* *}$ with a von Neumann algebra, for which the weak and $\sigma$-weak operator topologies coincide with the weak* topology, and the multiplication extends that of $\mathfrak{E}$. From $\S 2$, the second adjoint of the identity map of $\mathfrak{A}$ into $\mathfrak{C}$ is an isometry and a weak* homeomorphism of $\mathfrak{U}^{* *}$ onto $\overline{\mathfrak{A}}$, the weak operator closure of the $J$-algebra $\mathfrak{A} . \overline{\mathfrak{A}}$ is again a $J$-algebra (see [4]), hence a $J W$ algebra. As the isometry reduces to the identity on $\mathfrak{A}$, the resulting Jordan product on $\mathfrak{I}^{* *}$ extends that on $\mathfrak{A}$.

It is readily verified that the product on $\mathfrak{A}^{* *}$ coincides with both extensions of that on $\mathfrak{A}$ defined by Arens [1].

With the exception of the following paragraph, it will not be necessary to discuss the $\sigma$-weak operator topology. As it coincides with the weak operator topology on $\mathfrak{C}^{* *}$, the same is true on $\mathfrak{A}^{* *}$. In addition, any $\sigma$-weakly closed $J$-algebra $\mathfrak{A}$ is weakly closed. To see this we note that the unit ball $\mathfrak{U}_{1}$ is strongly, hence weakly dense in $(\overline{\mathfrak{A}})_{1}$. For, as has been indicated to us by Topping, the Kaplansky Density Theorem is valid for $J$-algebras (see, e.g., the proof in [5]). But $\mathfrak{A}_{1}$ is weakly compact, hence $\mathfrak{A}=\overline{\mathfrak{A}}$.

A representation $\phi$ of a $J$-algebra $\mathfrak{A}$ is a bounded linear map of $\mathfrak{A}$ into $\mathfrak{L}_{S A}$, the self-adjoint operators on some Hilbert space, satisfying $\phi(A \circ B)=\phi(A) \circ \phi(B)$. As $\phi: \mathfrak{A} \rightarrow \mathfrak{L}$ is continuous in the $\sigma\left(\mathfrak{A}, \mathfrak{U}^{*}\right)$ and $\sigma\left(\mathfrak{L}, \mathfrak{L}_{*}\right)$ topologies, the restriction map $\phi^{*}: \mathfrak{R}_{*} \rightarrow \mathfrak{A}^{*}$ is defined, and we obtain a $\sigma$-weakly continuous representation $\phi^{* *}: \mathfrak{A}^{* *} \rightarrow \mathfrak{L}$. Any $\sigma$-weakly continuous homomorphism of $J W$-algebras splits into an isometric isomorphism, and a zero map. As we shall not use this fact, and the proof is essentially the same as that for von Neumann algebras, we omit the details.

4. Ideal theory. A Jordan ideal $\mathfrak{\Im}$ in a $J$-algebra $\mathfrak{A}$ is a linear subspace of $\mathfrak{A}$ such that if $A \in \mathfrak{A}$ and $D \in \mathfrak{\Im}$, then $A \circ D \in \mathfrak{\Im}$. If $\mathfrak{\Im}$ is uniformly closed, the quotient space $\mathfrak{A} / \mathfrak{\Im}$ forms a (nonassociative) normed algebra in the usual way. The weak operator closure $\overline{\mathfrak{\Im}}$ is a Jordan ideal in $\overline{\mathfrak{A}}$. For if $A \in \mathfrak{A}$ and $D \in \overline{\mathfrak{J}}$, choose a net $D_{\alpha} \in \Im$ with $D_{\alpha} \rightarrow D$ weakly. Then $A \circ D_{\alpha} \in \Im$ and $A \circ D_{\alpha} \rightarrow A \circ D$, hence the latter is in $\overline{\mathfrak{J}}$. If $A \in \overline{\mathfrak{A}}$ and $D \in \overline{\mathfrak{J}}$, choose a net $A_{\alpha} \rightarrow A$. Then $A_{\alpha} \circ D \in \overline{\mathfrak{J}}$, $A_{\alpha} \circ D \rightarrow A \circ D$, and $A \circ D \in \bar{\Im}$.

Topping proved [4] that if $\mathfrak{A}$ is a $J W$-algebra, the lattice of projections in $\mathfrak{A}$ must be complete. Letting $E$ be the maximal projection in $\mathfrak{A}, A=A E=E A$ for all $A \in \mathfrak{A}$. He then pointed out that if $\mathfrak{\Im}$ is a weakly closed ideal in $\mathfrak{A}$, and $F$ is the maximal projection in $\mathfrak{x}$, then $\mathfrak{\Im}=\mathfrak{A} F$, and $F$ commutes with all the elements of $\mathfrak{A}$. 
THEOREM 2. Let $\Im$ be a-uniformly closed Jordan ideal in a J-algebra $\mathfrak{A}$. Then $\mathfrak{A} / \mathfrak{\Im}$ is isometrically isomorphic with a J-algebra, $\mathfrak{\Im}=[\mathfrak{\Im}] \cap \mathfrak{A}$, and [જ] is an ideal in $[\mathfrak{A}]$.

Proof. As we showed in the proof of Theorem $1, \mathfrak{A}^{* *}$ may be identified with the weak closure of $\mathfrak{A}$ in $\mathfrak{C}^{* *}$, where $\mathfrak{E}=[\mathfrak{A}]$. From above, the weak closure $\mathfrak{\Im}^{00}=\widetilde{\mathfrak{\Im}}$ is an ideal in $\overline{\mathfrak{A}}$. The maximal projection $F$ of $\bar{\Im}$ must lie in the center of $\mathfrak{C}^{* *}=[\mathfrak{A}]^{-}$.

Let $T$ be the canonical homomorphism of $\mathfrak{A}$ onto $\mathfrak{A} / \mathfrak{\Im}$. Then $T\left(\mathfrak{A}_{0}\right)=(\mathfrak{A} / \mathfrak{\Im})_{0}$ and $T^{* *}$ induces an isometry of $\mathfrak{A}^{* *} / \bar{\Im}$ onto $(\mathfrak{A} / \mathfrak{\Im})^{* *}$ (see $\left.\S 2\right)$. Composing the inverse of this map with the canonical isometry of $\mathfrak{A} / \mathfrak{\Im}$ into $(\mathfrak{A} / \mathfrak{\Im})^{* *}$, we obtain the map $A+\mathfrak{\Im} \rightarrow A+\overline{\mathfrak{F}}$, and conclude the latter is an isometric isomorphism of $\mathfrak{A} / \mathfrak{\Im}$ into $\overline{\mathfrak{U}} / \overline{\mathfrak{\Im}}$. On the other hand, the homomorphism $B+\overline{\mathfrak{\Im}} \rightarrow B(I-F)$ of $\overline{\mathfrak{A}} / \overline{\mathfrak{\Im}}$ onto $\overline{\mathfrak{A}}(I-F)$ is an isometry, as if $D \in \overline{\mathfrak{F}}$,

$$
\|B+D\|=\|B(I-F)+(B+D) F\|=\max [\|B(I-F)\|,\|(B+D) F\|],
$$

the von Neumann algebra $\mathfrak{C}^{* *}$ being isomorphic, hence isometric to $\mathfrak{C}^{* *} F$ $\oplus \mathfrak{C}^{* *}(I-F)$. Thus $A+\mathfrak{\Im} \rightarrow A(I-F)$ is an isometric isomorphism of $\mathfrak{A} / \mathfrak{\Im}$ into the $J W$-algebra $\overline{\mathfrak{A}}(I-F)$.

If $\mathfrak{\Im}^{\prime}$ is the weak* closure of $\mathfrak{\Im}$ in $\mathfrak{A}^{* *}$, we have from $\S 2$ that $\mathfrak{\Im}=\mathfrak{\Im}^{\prime} \cap \mathfrak{A}$. As the injection of $\mathfrak{A}^{* *}$ into $\mathfrak{C}^{* *}$ is a weak* homeomorphism, $\mathfrak{\Im}=\overline{\mathfrak{F}} \cap \mathfrak{A}$. As $A F=A$ for all $A \in \Im, C F=C$ for all $C \in[\Im]$, i.e., [अ] $\subseteq \mathfrak{C}^{* *} F$. Thus

$$
[\mathfrak{\Im}] \cap \mathfrak{A} \subseteq \mathfrak{\mho}^{* *} F \cap \overline{\mathfrak{A}}=\overline{\mathfrak{A}} F=\overline{\mathfrak{\Im}},
$$

and [अ] $\cap \mathfrak{A} \subseteq \Im$. The converse inclusion is trivial.

We next prove that $[\Im]^{-}=\mathfrak{C}^{* *} F$. It suffices to show that if $B \in \mathfrak{C}^{* *}=[\mathfrak{A}]^{-}$is such that $B=B F$, then $B \in[\Im]^{-} . B$ is a weak limit of finite linear combinations of terms of the form $A_{1} \cdots A_{n}$ with $A_{i} \in \mathfrak{A}$. Multiplying these sums by $F$, we may instead assume the $A_{i}$ lie in $\bar{\Im}=\overline{\mathfrak{U}} F$. Using the Kaplansky Density Theorem, we may select $A_{i}^{\alpha} \in \mathfrak{\Im}$ with $\left\|A_{i}^{\alpha}\right\| \leqq\left\|A_{i}\right\|$, and $A_{i}^{\alpha} \rightarrow A_{i}$ strongly. Thus $A_{1}^{\alpha} \cdots A_{n}^{\alpha} \rightarrow A_{1} \cdots A_{n}$ strongly, $A_{1} \cdots A_{n} \in[\Im]^{-}$, and $B \in[\Im]^{-}$.

It follows that $[\Im]^{-}$is an ideal in $\mathfrak{C}^{* *}$, and $[\Im]=[\Im]^{-} \cap \mathfrak{C}$ is an ideal in $\mathfrak{\complement}$.

\section{A characterization of Jordan ideals.}

THEOREM 3. Let $\Im$ be a subspace of a J-algebra $\mathfrak{A}$ with a multiplicative identity, or a uniformly closed subspace of an arbitrary J-algebra $\mathfrak{A}$. Then the following are equivalent:

(1) $\mathfrak{\Im}$ is a Jordan ideal in $\mathfrak{A}$.

(2) If $A \in \mathfrak{A}$ and $D \in \mathfrak{\Im}$, then $A D A \in \mathfrak{\Im}$.

Proof. That (1) always implies (2) follows from the equation

$$
A D A=\frac{1}{2}\left[(A(D A+A D)+(D A+A D) A)-\left(A^{2} D+D A^{2}\right)\right] .
$$


Conversely, say that (2) is satisfied and $E$ is a multiplicative identity for $\mathfrak{A}$. If $A \in \mathfrak{A}$ and $D \in \mathfrak{\Im}$, the $A \circ D \in \mathfrak{\Im}$ as

$$
A D+D A=(A+E) D(A+E)-A D A-D .
$$

If $\mathfrak{A}$ does not have an identity, let $E$ be the maximal projection in the $J W$-algebra $\mathfrak{A}^{* *}$. From the Kaplansky Density Theorem, there is a net $B_{\alpha} \in \mathfrak{A}$ with $\left\|B_{\alpha}\right\| \leqq 1$, converging strongly to $E$. It follows that

$$
\left(A+B_{\alpha}\right) D\left(A+B_{\alpha}\right)-A D A-D \rightarrow A D+D A
$$

in the strong, hence weak topologies, and $A D+D A \in \bar{\Im} \cap \mathfrak{A}$. As $\mathfrak{\Im}$ is convex and uniformly closed, we have from $\S 2, \bar{\Im} \cap \mathfrak{A}=\mathfrak{\Im}$.

\section{BIBLIOGRAPHY}

1. R. Arens, The adjoint of a bilinear operation, Proc. Amer. Math. Soc. 2 (1951), 839-848.

2. N. Bourbaki, Espaces vectoriels topologique, Actualités Sci. Indust. No. 1229, Hermann, Paris, 1955.

3. Z. Takeda, Conjugate spaces of operator algebras, Proc. Japan Acad. 30 (1954), 90-95.

4. D. Topping, Jordan algebras of self-adjoint operators, Mem. Amer. Math. Soc. No. 53 (1965), 48 pp.

5. J. Dixmier, Les algèbres d'opérateurs dans l'espace hilbertien, Gauthier-Villars, Paris, 1957.

University of Pennsylvania,

Philadelphia, Pennsylvania

UNIVERSITY OF OSLO,

OsLo, Norway 